\title{
Diversidade e Direitos Humanos na Atenção Primária à Saúde
}

\author{
Diversity and Human Rights in Primary Health Care
}

\section{Diversidad y derechos humanos en la atención primaria de salud}

\author{
Thiago Dias Sarti@ ${ }^{1}$, Denize Ornelas Pereira Salvador de Oliveira ${ }^{2}$,Patrícia Sampaio Chueiri ${ }^{3}{ }^{2}$ Leonardo Ferreira Fontenelle ${ }^{4}$ \\ ${ }^{1}$ Universidade Federal do Espírito Santo (UFES). Vitória, ES, Brasil. \\ ${ }^{2}$ Secretaria de Saúde de São Bernardo do Campo. São Bernardo do Campo, SP, Brasil. \\ ${ }^{3}$ Escola de Medicina da Faculdade Israelita de Ciências da Saúde Albert Einstein. São Paulo, SP, Brasil. \\ ${ }^{4}$ Universidade Vila Velha (UVV). Vila Velha, ES, Brasil.
}

\section{Resumo}

Neste editorial, apresenta-se o resultado da chamada pública de artigos que compõem o especial "Diversidade e Direitos Humanos na Atenção Primária à Saúde". A situação dos direitos humanos no Brasil é preocupante, existindo profundas desigualdades no exercício pleno da cidadania entre grupos sociais, o que inclui o acesso a serviços de saúde e manutenção de níveis de saúde adequados e justos. Questões raciais, de gênero, sexualidade, diversidade, violência e direitos humanos ganham cada vez mais importância no contexto da atenção primária à saúde (APS) e da Medicina de Família e Comunidade (MFC). Os trabalhos publicados neste especial apontam para a importância de pensarmos a organização de uma APS interseccional e uma prática em saúde abrangente, acolhedora, humanizada, resolutiva e culturalmente e socialmente orientada. Sugere-se que a MFC precisa produzir reflexões teóricas mais robustas para subsidiar o cuidado a pessoas em graves situações de vulnerabilidade e sofrimento. Aponta-se ainda para a necessária equidade de gênero, etnia e territorial na composição do corpo editorial da RBMFC. Espera-se que o conteúdo deste especial apoie a reflexão e a construção de práticas e de políticas que consigam enfrentar o quadro das profundas e diversas desigualdades sociais do país, e que no futuro possamos contar com uma maior produção de conhecimento neste campo.

Palavras-chave: Atenção Primária à Saúde; Cuidado Centrado no Paciente; Direitos Humanos; Diversidade Cultural; Disparidades nos Níveis de Saúde

\begin{abstract}
This editorial presents the result of the public call for articles that make up the special "Diversity and Human Rights in Primary Health Care". The human rights situation in Brazil is worrying, with profound inequities in the full exercise of citizenship among social groups, including access to health services and maintenance of adequate and fair health levels. Racial, gender, sexuality, diversity, violence and human rights are becoming increasingly important in the context of primary health care $(\mathrm{PHC})$ and Family and Community Medicine.The works published in this special point to the importance of thinking about the organization of an intersectional PHC and a comprehensive, welcoming, humanized, resolute and culturally and socially oriented health practice. Family and Community Medicine should produce more robust theoretical reflections to support care for people in severe situations of vulnerability and suffering. At the same time, the composition of RBMFC's editorial board should have greater equity in gender, ethnicity and geography. We hope the content of this special will support the reflection on and construction of practices and policies able to face the deep and diverse social inequalities of the country, and that in the future we can count on a greater knowledge production in this field.
\end{abstract}

Keywords: Primary Health Care; Patient-Centered Care; Human Rights; Cultural Diversity; Health Status Disparities

Como citar: Sarti TD, Oliveira DOPS, Chueiri PS, Fontenelle LF. Diversidade e Direitos Humanos na Atenção Primária à Saúde. Rev Bras Med Fam Comunidade. 2019;14(41):2259. https://doi.org/10.5712/rbmfc14(41)2259

\author{
Autor correspondente: \\ Thiago Dias Sarti. \\ E-mail: thiagosarti@yahoo.com.br \\ Fonte de financiamento: \\ declaram não haver. \\ Parecer CEP: \\ não se aplica.
}

Procedência e revisão por pares:

Não revisado por pares.

Recebido em: 23/10/2019.

Aprovado em: 23/10/2019. 


\section{Resumen}

Este editorial presenta el resultado de la convocatoria pública de artículos que componen el especial "Diversidad y derechos humanos en la atención primaria de salud". La situación de los derechos humanos en Brasil es preocupante, con profundas desigualdades en el ejercicio pleno de la ciudadanía entre los grupos sociales, incluido el acceso a los servicios de salud y el mantenimiento de niveles de salud adecuados y justos. Las cuestiones raciales, de género, sexualidad, diversidad, violencia y derechos humanos son cada vez más importantes en el contexto de la atención primaria de salud (APS) y la medicina de familia y comunidad (MFC). Los trabajos publicados en este especial señalan la importancia de pensar en la organización de una APS interseccional y una práctica de salud integral, acogedora, humanizada, resuelta y cultural y socialmente orientada. Se sugiere que la MFC necesita producir reflexiones teóricas más sólidas para apoyar la atención de personas en situaciones graves de vulnerabilidad y sufrimiento. También se señala la necesidad de igualdad de género, etnia y territorial en la composición del consejo editorial de RBMFC. Se espera que el contenido de este especial respalde la reflexión y la construcción de prácticas y políticas que puedan hacer frente a la imagen de las profundas y diversas desigualdades sociales del país, y que en el futuro podamos contar con una mayor producción de conocimiento en este campo.

Palabras clave: Atención Primaria de Salud; Atención Dirigida al Paciente; Derechos Humanos; Diversidad Cultural; Disparidades en el Estado de Salud

A Revista Brasileira de Medicina de Família e Comunidade (RBMFC) tem o prazer de apresentar a suas leitoras e leitores e a toda a comunidade acadêmica e de profissionais de saúde os artigos que compõem o Especial "Diversidade e Direitos Humanos na Atenção Primária à Saúde".

Aideia desta chamada pública de artigos surgiu da crescente importância que as questões raciais, de gênero, sexualidade, diversidade, violência e direitos humanos ganham no contexto da atenção primária à saúde (APS) e da Medicina de Família e Comunidade (MFC). A publicação de artigos com tais temáticas visa criar mais um espaço de debate entre os MFCs e demais interessados, a fim de visibilizar e amplificar reflexões que tenham potência para melhorar o cuidado de pessoas em situação de vulnerabilidade naAPS.

Os artigos desta edição especial chegam em um momento muito rico para a especialidade, com a oficialização pela Sociedade Brasileira de Medicina de Família e Comunidade (SBMFC) dos seguintes grupos de trabalho: Mulheres na Medicina de Família e Comunidade; Gênero, Sexualidade, Diversidade e Direitos; e Saúde da População Negra. E cada vez mais os temas desta edição compõem as diferentes atividades dos nossos congressos nacionais, regionais e locais, graças ao trabalho de médicas e médicos de família e comunidade que dedicam parte significativa de seu afeto, cuidado e energia a refletir, produzir e ensinar práticas de saúde contextualizadas, abrangentes, significativas, acolhedoras e centradas no sofrimento das pessoas. Todos estes espaços e sujeitos contribuem para enriquecer o arcabouço teórico e prático da MFC e da APS, incorporando saberes, práticas e ampliando relações com movimentos sociais feministas, negros, LGBT (lésbicas, gays, bissexuais e pessoas trans), dentre outros.

A situação dos direitos humanos no Brasil é preocupante, existindo profundas desigualdades no exercício pleno da cidadania entre grupos sociais. ${ }^{1,2}$ Há anos, o país apresenta elevadas taxas de mortes violentas de mulheres, pessoas transexuais e de jovens negros, principalmente quando são pobres e têm baixa escolaridade.$^{1-3} \mathrm{~A}$ realidade da população carcerária ainda se traduz em superlotação, violência, $\mathrm{e}$ precárias condições de saúde. ${ }^{1}$

Grande parte dos trabalhadores do país estão na informalidade. ${ }^{4}$ Mulheres continuam a ter salários menores que homens para serviços semelhantes, inclusive na Medicina, ${ }^{5}$ enquanto assistimos retrocessos em relação a conquistas de direitos no campo da Saúde Sexual e Reprodutiva. ${ }^{6}$ Aproximadamente um terço das pessoas com 19 a 24 anos de idade não concluem o ensino médio, já as pessoas negras têm indicadores de acesso e permanência na escola significativamente piores que pessoas brancas. ${ }^{7}$ Imigrantes internacionais, problema crescente no país, vivem o sofrimento da mudança forçada para 
outro país, enfrentando dificuldades de acesso aos serviços públicos, incluindo os de saúde. ${ }^{8}$ E persistem iniquidades em saúde quando se considera cor de pele da pessoa, que se traduzem em pior qualidade de vida e maior morbimortalidade de negras e negros. ${ }^{9}$

Este quadro deve ser enfrentado com políticas públicas que visem combater a desigualdade, construídas a partir das melhores evidências disponíveis, pois são elas que têm o maior potencial de mudança da realidade. ${ }^{10}$

A chamada pública recebeu número significativo de artigos, mais do que o esperado, em especial de relatos de experiência. Tais relatos em geral versavam sobre grupos operativos com usuários e capacitações com profissionais de saúde em unidades básicas de saúde, atividades educativas em escolas de ensino fundamental e médio, eventos e atividades didáticas em nível de graduação em escolas médicas, formações com médicos residentes e MFCs em geral, relatos de casos de pessoas atendidas em serviços cuja abordagem explorou as principais ferramentas da APS e da MFC, etc.

Infelizmente, a maior parte destes trabalhos não atendiam aos requisitos para publicação, mas este conjunto de experiências sinaliza para um rico movimento de debates, diagnósticos e ações que ocorrem nos mais diversos cenários, potencializando sujeitos para a prática de um cuidado humanizado e centrado na vida das pessoas. Estas iniciativas abrem possibilidades de construção coletiva de saberes e práticas que poderão produzir novos sujeitos para a saúde capazes de refletir sobre práticas sociais cristalizadas que em alguma medida influenciam negativamente a relação com as pessoas que procuram os serviçosde saúde.

No entanto, estes relatos de experiência também evidenciaram a necessidade de maior aprofundamento teórico e elaboração metodológica das ações. É fundamental incorporar conceitos essenciais às práticas de saúde junto a pessoas vítimas de racismo estrutural, homofobia, transfobia, machismo e misoginia, preconceitos de distintas ordens, violência, discriminação social e econômica.

Não é trivial lidar com situações de vulnerabilidade social que surgem no cotidiano dos serviços de saúde, exigindo atitudes e habilidades de comunicação orientadas culturalmente e socialmente a populações que em sua maioria vivem em situações bem distintas daquelas dos profissionais de saúde. ${ }^{11}$ Muitos relatos enviados para esta chamada limitavam-se a ações pontuais com pressupostos pedagógicos verticalizados e em alguma medida normativos, elitista e moralizantes.

Contudo, também se viu experiências que partiam dos saberes, vozes e visões de mundo dos sujeitos em cena, sendo possível observar uma desnaturalização de relações sociais e imagens do outro que produzem formas de estar em equipe e cuidar das pessoas mais potentes e horizontais. Tais experiências apontam para uma APS interseccional ${ }^{12}$ que consegue perceber e cuidar, por exemplo, de uma mulher trans negra pobre periférica com todas as marcas existenciais que isso pode trazer para as pessoas e encontram eco nas palavras de Bell Hooks ${ }^{13}$ (p. 42):

[...] para as mulheres de grupos oprimidos que têm reprimido tantos sentimentos - desespero, fúria, angústia - que não falam, como escreve o poeta Audre Lorde, "pelo medo de nossas palavras não serem ouvidas nem bem-vindas", encontrar a voz é um ato de resistência. Falar se torna tanto uma forma de se engajar em uma autotransformação ativa quanto um rito de passagem quando alguém deixa de ser objeto e se transforma em sujeito. Apenas como sujeitos é que podemos falar. Como objetos permanecemos sem voz - e nossos seres, definidos e interpretados pelos outros. 
Os trabalhos publicados nesse especial apontam para a importância de pensarmos a organização de uma APS e uma prática em saúde abrangente, acolhedora, humanizada, resolutiva e culturalmente e socialmente orientada. Como mostram Pereira e Chazan, ${ }^{14}$ mesmo em um nível de atenção que deveria

se caracterizar pela facilidade de acesso como é a APS, existem barreiras significativas que afetam distintamente a capacidade de grupos sociais encontrarem uma fonte permanente de cuidados integrais.

Diversos estudos já mostraram também que a relação interpessoal se molda ao perfil dos profissionais e dos usuários, podendo reduzir a qualidade da interação e, consequentemente, a qualidade da atenção ofertada. ${ }^{11} \mathrm{Em}$ um país diverso e desigual como o Brasil, é papel da APS enxergar esses problemas, contribuindo substancialmente para a redução das iniquidades em saúde que ainda marcam o cenário epidemiológico e existencial da população. ${ }^{15}$

Nesta chamada, incentivados pelas discussões trazidas pelo GT Mulheres na MFC, buscamos incentivar autoras e autores a adotarem as Diretrizes SAGER (Sex and Gender Equity in Research) ${ }^{16}$ para auxiliar na construção sistemática dos relatos das informações sobre sexo e gênero na metodologia dos estudos, na análise e apresentação dos dados e na discussão dos resultados.

Possivelmente, nosso êxito nesta implantação ainda esteja longe do ideal, mas é preocupação central da RBMFC construir práticas editoriais e de divulgação científica que respeitem a equidade não apenas de gênero, mas também de raça/cor e territorial. Infelizmente, isso ainda não está consolidado no corpo editorial da revista (por exemplo, dos atuais 2 editores chefe, 7 membros do conselho editorial e 18 editores associados, temos nenhuma, 1 e 6 mulheres, respectivamente, embora tenha-se buscado contemplar todas as regiões do país, sem, contudo, conseguir eliminar a concentração de pessoas das regiões Sul e Sudeste) e no cadastro de avaliadores e autores.

Além disso, em recente trabalho, Fontenelle et al. ${ }^{17}$ observaram que a probabilidade das mulheres MFC adquirirem os títulos de mestrado e/ou doutorado é inferior à dos homens, o que é um padrão diferente em relação às demais áreas, evidenciando um grave problema inclusive ético que merece ser melhor compreendido e solucionado. É neste sentido que concordamos com o grupo editorial de Lancet, ${ }^{18}$ uma das principais revistas científicas do mundo, ao conclamar por mudanças institucionais em todo o aparato científico, incluindo o corpo editorial das revistas acadêmicas, que reduzam as iníquas disparidades de gênero, étnicas e sociais. Diversidade em ciência é central para uma produção acadêmica mais relevante e de maior impacto social.

Por fim, esperamos que o conteúdo desta edição apoie a reflexão e a construção de práticas e de políticas que consigam enfrentar o quadro de desigualdade do país, e que no futuro possamos contar com uma maior produção de conhecimento neste campo, como parte do papel que temos na nossa especialidade, no combate e redução das iniquidades em saúde.

\section{Conflito de interesses}

Declaram não haver. 


\section{Referências}

1. Human Rights Watch. 29th annual World Report. New York: Seven Stories Press; 2019.

2. Ministério da Economia (BR), Instituto de Pesquisa Econômica Aplicada, Fórum Brasileiro de Segurança Pública. Atlas da violência 2019. Brasília: Rio de Janeiro/São Paulo: Instituto de Pesquisa Econômica Aplicada; Fórum Brasileiro de Segurança Pública; 2019.

3. Queiroga L. Brasil segue no primeiro lugar do ranking de assassinatos de transexuais. O Globo. 2018 Nov 14 ; Sec. Sociedade.

4. Ministério da Economia (BR), Instituto Brasileiro de Geografia e Estatística. (IBGE). Pesquisa Nacional por Amostra de Domicílios Contínua

5. PNAD Contínua [Internet]. 2019. [acesso 2019 Out 22]. Disponível em: https://www.ibge.gov.br/estatisticas/sociais/trabalho/17270pnad-continua.html?=\&t=0-que-e

6. Scheffer MC, Cassenote AJF. A feminização da medicina no Brasil. Rev Bioét. 2013;21(2):268-77. https://doi.org/10.1590/S198380422013000200010

7. Zanatta LF, Grein MI, Álvarez-Dardet C, Moraes SP, Brêtas JRS, Ruiz-Cantero MT, et al. Igualdade de gênero: por que o Brasil vive retrocessos? Cad Saúde Pública. 2016;32(8):e00089616. https://doi.org/10.1590/0102-311X00089616

8. Saldaña P. 4 em cada 10 jovens negros não terminaram o ensino médio: dados do IBGE mostram que abandono escolar atinge mais

9. população negra. Folha de São Paulo. 2019 Set 1; Sec. Cotidiano.

10.Martin D, Goldberg A, Silveira C. Imigração, refúgio e saúde: perspectivas de análise sociocultural. Saúde Soc. 2018;27(1):26-36. https://doi.org/10.1590/s0104-12902018170870

11. Hone T, Rasella D, Barreto ML, Majeed A, Millett C. Association between expansion of primary healthcare and racial inequalities in mortality amenable to primary care in Brazil: A national longitudinal analysis. PLoS Med. 2017;14(5):e1002306. https://doi.org/10.1371/ journal.pmed.1002306

12.Marmot M. The Health Gap: the challenge of an unequal world. New York: Bloomsbury Press; 2015. https://doi.org/10.1016/S01406736(15)00150-6

13.Gusso G, Lopes JMC. Tratado da Medicina da Família e Comunidade. 2ª ed. Porto Alegre: Artmed; 2019.

14.Akotirene C. Interseccionalidade. São Paulo: Pólen Livros; 2019.

15. Hooks B. Erguer a voz: pensar como feminista, pensar como negra. São Paulo: Editora Elefante; 2018.

16.Pereira LBC, Chazan ACS. O Acesso das Pessoas Transexuais e Travestis à Atenção Primária à Saúde: uma revisão integrativa. Rev Bras Med Fam Comunidade. 2019;14(41):1795. https://doi.org/10.5712/rbmfc14(41)1795

17.Barreto ML. Desigualdades em Saúde: uma perspectiva global. Ciênc Saúde Coletiva. 2017;22(7):2097-108. https://doi.org/10.1590/1413- 81232017227.02742017

18. Heidari S, Babor TF, De Castro P, Tort S, Curno M. Sex and Gender Equity in Research: rationale for the SAGER guidelines and recommended use. Res Integr Peer Rev. 2016;1:2. https://doi.org/10.1186/s41073-016-0007-6

19.Fontenelle LF, Rossi SV, Oliveira MHM, Brandão DJ, Sarti TD. Postgraduate education among family and community physicians in Brazil: the Trajetórias MFC project. medRxiv. 2019;19005744. https://doi.org/10.1101/19005744

20.The Editors of the Lancet Group. The Lancet Group's commitments to gender equity and diversity. Lancet. 2019;394(10197):452-3. https://doi.org/10.1016/S0140-6736(19)31797-0 